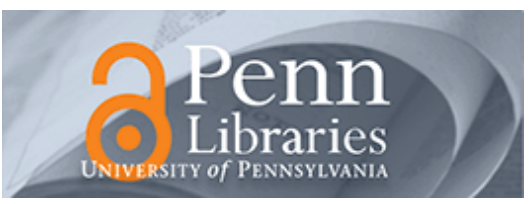

University of Pennsylvania

ScholarlyCommons

8-31-2012

\title{
Analysis of Slotted ALOHA with Multipacket Messages in Clustered Surveillance Networks
}

\author{
Soumya Sen \\ Princeton University \\ David J. Dorsey \\ Lockheed Martin \\ Roch A. Guérin \\ University of Pennsylvania, guerin@acm.org \\ Mung Chiang \\ Princeton University
}

Follow this and additional works at: https://repository.upenn.edu/ese_papers

Part of the Digital Communications and Networking Commons, and the OS and Networks Commons

\section{Recommended Citation}

Soumya Sen, David J. Dorsey, Roch A. Guérin, and Mung Chiang, "Analysis of Slotted ALOHA with Multipacket Messages in Clustered Surveillance Networks", . August 2012.

2012 IEEE Military Communications Conference (Milcom) - Unclassified Sessions October 2012, Orlando, FL

(c) 2012 IEEE. Personal use of this material is permitted. Permission from IEEE must be obtained for all other uses, in any current or future media, including reprinting/republishing this material for advertising or promotional purposes, creating new collective works, for resale or redistribution to servers or lists, or reuse of any copyrighted component of this work in other works.

This paper is posted at ScholarlyCommons. https://repository.upenn.edu/ese_papers/613

For more information, please contact repository@pobox.upenn.edu. 


\title{
Analysis of Slotted ALOHA with Multipacket Messages in Clustered Surveillance Networks
}

\begin{abstract}
This work presents an analysis of a cluster of finite population of low cost sensor nodes operating in a p-persistent S-Aloha framework with multipacket messages. Using this analytical framework, we consider the issue of partitioning the nodes and available frequencies into groups so as to maximize the system throughput. Assigning the nodes and frequencies into "groups" is important because the size of the group impacts the tradeoff between the benefits of frequency diversity and the cost of collision on the shared medium imposed by the nodes in a group. We study this tradeoff through analytical and numerical results and show how the correct choice of group sizes can vary depending on various factors like the ratio of nodes to frequencies and the overall system load.
\end{abstract}

\section{Keywords}

Aloha, multi-user, multi-frequency, grouping

\section{Disciplines \\ Digital Communications and Networking | OS and Networks}

\section{Comments}

2012 IEEE Military Communications Conference (Milcom) - Unclassified Sessions

October 2012, Orlando, FL

(C) 2012 IEEE. Personal use of this material is permitted. Permission from IEEE must be obtained for all other uses, in any current or future media, including reprinting/republishing this material for advertising or promotional purposes, creating new collective works, for resale or redistribution to servers or lists, or reuse of any copyrighted component of this work in other works. 


\title{
Analysis of Slotted ALOHA with Multipacket Messages in Clustered Surveillance Networks
}

\author{
Soumya Sen*, David J. Dorsey ${ }^{\dagger}$, Roch Guérin ${ }^{\ddagger}$, and Mung Chiang* \\ *Electrical Engineering, Princeton University, Princeton, NJ 08544 \\ Email: \{soumyas, chiangm\}@ princeton.edu \\ $\dagger$ Lockheed Martin ATL, Cherry Hill, NJ, USA \\ Email: david.j.dorsey@ Imco.com \\ ${ }_{\ddagger}^{\ddagger}$ Electrical Engineering, University of Pennsylvania, Philadelphia, PA, USA \\ Email: guerin@ee.upenn.edu
}

\begin{abstract}
This work presents an analysis of a cluster of finite population of low cost sensor nodes operating in a $p$ persistent S-Aloha framework with multipacket messages. Using this analytical framework, we consider the issue of partitioning the nodes and available frequencies into groups so as to maximize the system throughput. Assigning the nodes and frequencies into "groups" is important because the size of the group impacts the tradeoff between the benefits of frequency diversity and the cost of collision on the shared medium imposed by the nodes in a group. We study this tradeoff through analytical and numerical results and show how the correct choice of group sizes can vary depending on various factors like the ratio of nodes to frequencies and the overall system load.
\end{abstract}

\section{INTRODUCTION}

The growing availability of affordable wireless devices is creating new opportunities for large scale distributed sensing and surveillance for military operations. High density deployment of these low cost COTS-based devices as Points of Presence (POPs) close to the targets (devices or facilities) can be used to improve situational awareness, collaborative monitoring, threat recognition, and Electronic Warfare Surveillance (ES). These POPs can monitor and report in a distributed manner to a rendezvous node or a cluster-head with SATCOM capabilities, which then aggregates, filters, and relays the information to a remote operation command center (e.g., EWO in a prophet vehicle). An example of such a deployment scenario is shown in Figure 1.

But since these POPs operate in an uncoordinated (decentralized) manner to send reports to the cluster-head, they contend for the access channels, resulting in transmission collisions with other POPs. In such scenarios with shared medium, various techniques can be used to reduce collisions. For example, the Ethernet uses CSMA-CD as a MAC protocol, while 802.11 wireless LAN uses CSMA-CA. But in wireless communication settings, collision detection (CD) is expensive and collision avoidance through carrier sensing is often difficult for high-density, low-cost devices. An alternative, lightweight MAC protocol that has been often used in scenarios with such "hidden-node" problem is the Slotted Aloha (S-Aloha). S-Aloha is a decentralized MAC protocol without carrier sensing in which transmitting devices are restricted to sending packets within discrete time slots. Aloha channels

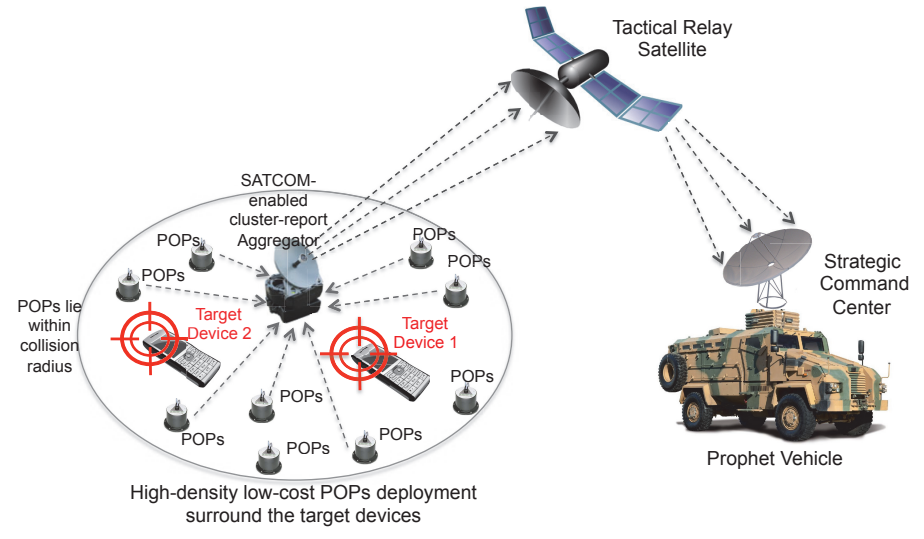

Fig. 1. Surveillance Network Scenario

were used in a limited way in $1 \mathrm{G}$ mobile phones for signaling and controlling, and since 1990s for SMS in 2G. In early $2000 \mathrm{~s}$, additional Aloha channels were added to $2.5 \mathrm{G}$ and $3 \mathrm{G}$ mobile phones with the introduction of GPRS. S-Aloha is also widely used in many underwater sensor networks, satellite networks, wireless radios etc. Therefore, the simple S-Aloha protocol is a likely protocol for the COTS sensor network scenario of Figure 1.

Previous works have analyzed Aloha and several of its variants for their throughput and stability, as discussed in Section II. In this work, we consider a $p$-persistent S-Aloha model [2] with saturated arrival, in which a node backs off with a positive probability after each message transmission. This back off mechanism helps to prevent channel capture by any one nodes. Our work extends this framework to a setting with multi-packet messages with a finite number of users and a finite set of channels (frequency sets).

But more importantly, the closed form expressions we derive for throughput in this generic setting allow us to answer the practical question of efficient resource partitioning: How should we "group" the POPs into MAC subnets and assign frequency sets to them so as to improve the system throughput? For example, if we have $M=8$ nodes and $F=4$ frequencies to allocate, is it better to form: (a) 4 groups with two nodes and only one frequency assigned to each group, or (b) 2 larger groups with four nodes and two frequencies assigned to each 
group, such that the four nodes can transmit on any of the two frequencies, or (c) one large group with all eight nodes sharing all the four frequencies? At the heart of this question is the tradeoff between the impact of increase in channel diversity from more available frequencies and the potential for a greater contention among accessing nodes. Understanding this tradeoff requires analyzing the throughput of such a multipacket, finite user, S-Aloha system for a range of possible "grouping" or clustering of sensor nodes and frequencies.

The key features and contributions of this work are:

- We model a deployment scenario of low cost sensor devices that operate in a basic random access mode and study the issue of partitioning these nodes and available frequencies to maximize throughput.

- Our analysis provides closed form expressions for the throughput of multi-packet, p-persistent S-Aloha system with finite number of nodes and frequencies.

- Our results provide guidelines in network planning and resource allocation by illustrating that the right choice of clustering or "grouping" nodes and frequencies will vary depending on factors like the system load.

The paper is organized as follows: Section II discusses the related literature, Section III introduces the model and Section IV analyzes the throughput of the system for different scenarios, the results and numerical examples are reported in Section V, followed by the conclusion in Section VI.

\section{RELATED LiterATURE}

Aloha is a popular low complexity random access protocol [4] that has been extensively analyzed. Ferguson [5] and Bellini [6] considered the performance of pure (unslotted) Aloha with infinite user population and variable packet length. [7] considers the performance of S-Aloha system with multipacket buffers. Raychaudhuri [1] extended these early works to S-Aloha systems in which messages generated by users consists of multiple packets instead of only one.

Another variation of slotted Aloha, known as $p$-persistent SAloha, considered in this work, uses a persistence parameter, $p$, to determine the probability that a node transmits a packet in a slot [2]. Our work extends this line of investigation by computing throughput for multi-packet messages with a finite population and finite frequency set. Additionally, we use our formulation to address the issue of network resource partitioning and its impact on throughput.

Researchers have also focused on stability of the Aloha [9], but this issue is not pertinent to our study because our model, like that of [3], [11], considers a saturated arrival scenario. [3] proposes a generalized S-Aloha in which nodes back off with a probability that depends on whether the previous packet transmission has been successful. But unlike [3], the low-cost nodes of our setting typically lack expensive CD capability and ACK mechanisms (due to long RTTs often arising in such scenarios), and hence, are assumed to back off with a probability $p$ to avoid channel capture issues.

Performance analysis of strategic behavior in Aloha [10] and game theoretic formulations [11] have also been considered.

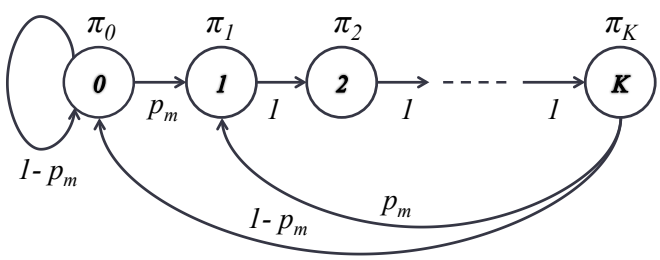

Fig. 2. Markov chain of the S-Aloha model considered

Although our present work does not involve strategic game formulation since we consider only low cost sensor nodes with limited computational capacity, it is a topic of potential interest for future work.

\section{MODEL}

In this section, we introduce the system model and its parameters. We consider a cluster with $M$ transmitting nodes (POPs) and $F$ frequencies to share. Some key feature of the scenario considered are:

1) A node (POP) always has "messages" to transmit, i.e., saturated arrivals (i.e. elastic transfer).

2) These "messages" sent by the nodes are multi-packet messages, each constituted of $k$ number of packets.

3) All the $k$ packets in the message are transmitted back to back, one in each time slot.

4) In each transmitting slot, a node can choose any one of the $F$ frequencies uniformly at random to transmit a packet of the message.

5) Collision occurs when two or more nodes transmit message packets simultaneously on the same frequency.

Since the focus of this work is on performance measured in terms of attainable throughput, and not on stability, we follow [3] in considering a system in a saturated arrival mode. Classical stability analysis is not applicable to this model because the system is assumed to operate in a saturated arrival, and hence throughput is the primary performance metric considered.

Like the traditional Aloha protocol, we consider only "no capture" setting, i.e., collided messages are lost. Additionally, we assume that the low-cost nodes lack CD capabilities (e.g., it is difficult for radios to listen for interference while actively transmitting) and long RTT for ACKs (as in satellite links) and additional buffering needs render link layer packet-by-packet ACKs or selective ARQ unviable (i.e. message retransmissions are taken care of at higher layers of the protocol stack). In other words, if any of the $k$ back-to-back packets from two message transmissions collide, both the collided message are assumed to be lost. We also consider perfect channel condition in this model, but extension to an i.i.d. settings is fairly straightforward. Earlier research has highlighted the potential benefits that channel diversity can provide due to randomization over the access channels [12]. In practice, such fast slot-to-slot frequency switching can be enabled by using two network interface cards (NICs) or frequency agile transmitters.

The $p$-persistent Slotted Aloha protocol considered in this work has the following features: 
- If a node $m$ has a message to send, then it begins to transmit the first packet in the next slot with a probability $p_{m}$, followed by the rest of the $k-1$ packets back to back, one in each time slot.

- Once a message has been transmitted, the node transmits the first packet of the next message in the next slot with a probability $p_{m}$ or backs off with a probability $1-p_{m}$.

Given that the nodes of the system are uncoordinated and independent of each other, the resulting Markov chain for an individual node is shown in Figure 2. The states of a given node $m$, are denoted by $i \in\{0, \ldots, k\}$, with stationary probabilities of $\pi_{i}$, where $\pi_{0}$ corresponds to an "idle" state and others being packet transmitting states. When a node is in $k$-th state, the node is sending the last packet of a message, followed by which it can back-off into an idle or inactive state with probability $1-p_{m}$ or transition to state 1 with probability of $p_{m}$ to send the first packet of the next message. The steady state probabilities are therefore given by:

$\pi_{0}=\frac{1-p_{m}}{1-p_{m}(1-k)}, \quad \pi_{i}=\frac{p_{m}}{1-p_{m}(1-k)} \forall i \in\{1, \ldots, k\}$

A similar Markov chain can be constructed when we define each state by a tuple $(i, j)$, where $i=\{0, \ldots, k\}$ denotes the packet transmission state and $j=\{1, \ldots, F\}$ denotes the frequency of transmission. Given that the frequency hopping is done uniformly across the set of frequencies, we obtain the following expressions:

$$
\begin{aligned}
\pi_{0} & =\frac{1-p_{m}}{1-p_{m}(1-k)} \\
\pi_{i j} & =\frac{p_{m}}{\left[1-p_{m}(1-k)\right] F} \forall 0<i \leq k, 1 \leq j \leq F
\end{aligned}
$$

We use these expressions in Section IV to calculate successful message transmission probabilities in various scenarios.

\section{ANALYSIS}

In this section, we first derive the throughput expressions for a homogeneous system in which each node has the same message transmission probability of $p=p_{m}$. Then we extend the results for a scenario with heterogeneous system with distinct $p_{m}$ for each node.

\section{A. Homogeneous Nodes}

1) Single Frequency Case: When a finite number of nodes, $M$, share a single frequency to transmit $k$-packet length messages using described $p$-persistent S-Aloha protocol, the message transmission is successful if no other node was active at during the duration of this transmission, that is, the unconditional success probability $P_{s}$ for a given node is:

$$
P_{s}=\pi_{1} \pi_{0}^{M-1}\left((1-p)^{M-1}\right)^{k-1}=\frac{p(1-p)^{(M-1) k}}{[1-p(1-k)]^{M-1}}
$$

where $\pi_{0}$ and $\pi_{1}$ are given by Eqn. (1).

For the special case of $k=1$, i.e., single packet messages, this probability is $P_{s}=p(1-p)^{M-1}$.

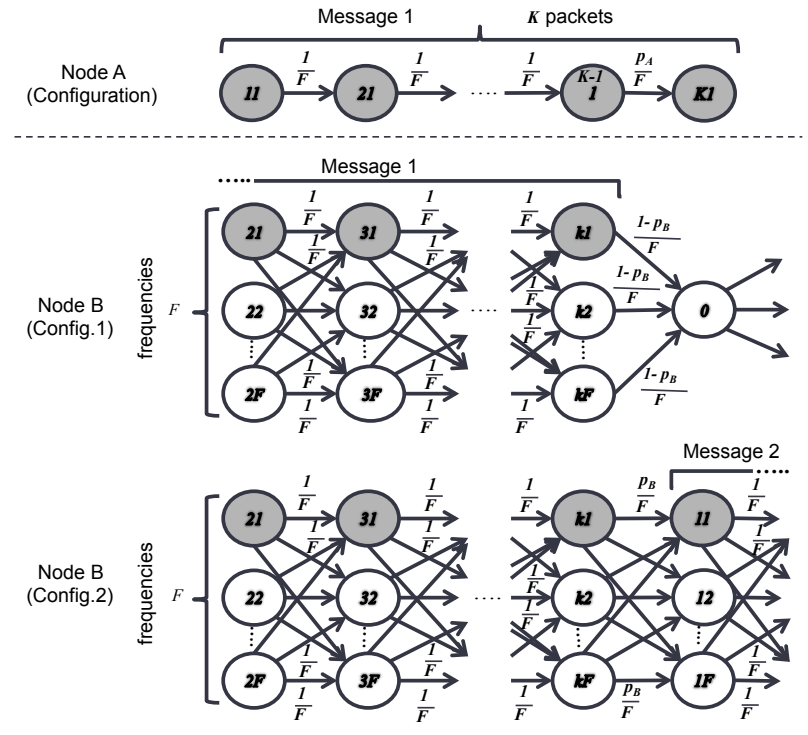

Fig. 3. Markov chains of possible configurations when Node B is already transmitting the second packet

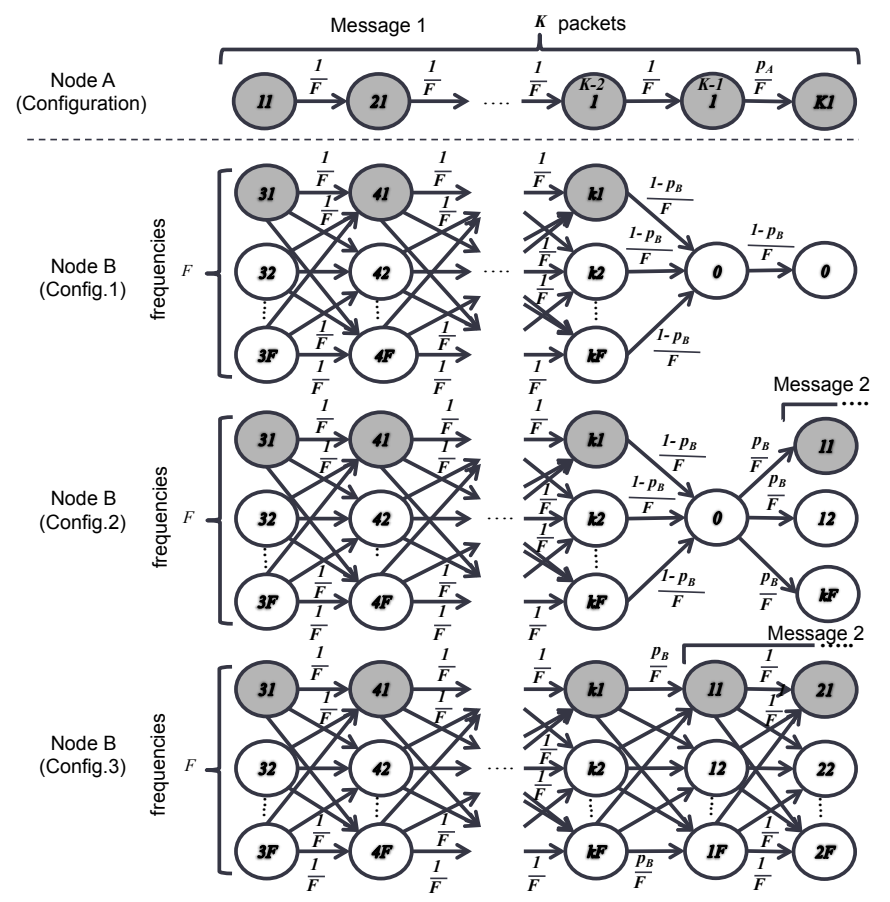

Fig. 4. Markov chains of possible configurations when Node B is already transmitting the third packet

2) Multiple Frequency Case: In the case of multiple frequencies, deriving the probability of a successful message transmission is particularly difficult because of the possibility of messages from any two nodes colliding at one or more of the $k$ time slots. This difficulty arises because a particular node's throughput depends on: (1) whether each of the other nodes were active or inactive, either entirely or partially, during the duration of this node's message transmission, and (2) whether any of the actively transmitting nodes chose the same frequency in any of the time slots as this particular node during its message transmission. 
To obtain general expressions for the probability of a successful transmission, we consider two nodes, $A$ and $B$. Without loss of generality, let us assume that Node $A$ becomes active at some time $t=1$ and transmits the $k$ packets of a message, one in each consecutive slots, all on frequency 1. Note that this is only one possible realization, the node could have switched over to any other frequency at any time slot with probability of $1 / F$. Next, assume that Node $B$ was inactive at time $t=1$, but it could become active in any of these $k$ time slots with probability $p$ and choose the same frequency as Node $A$ with probability of $1 / F$ in any time slot. It can be shown that the conditional probability that Node $A$ 's message does not collide with Node $B$, given that Node $A$ became active at time slot 1 is

$$
\pi_{0}\left[\sum_{n=1}^{k-1} p(1-p)^{n}\left(1-\frac{1}{F}\right)^{k-n}+(1-p)^{k-1}\right]
$$

Similarly, we now consider the case where both Nodes $A$ and $B$ start simultaneously transmitting their first packet of a message at $t=1$. Let $f_{A(i)}$ denote the frequency on which Node $A$ is transmitting its packet in the $(i=1)$-th time slot, and $\pi_{1 j}$ be the initial state Node $B$, where $j \neq f_{A(i)}$ so that its first packet did not collide on the frequency used by Node $A$. We then have the conditional probability that Node $A$ 's message does not collide with Node $B$ 's on any of the next $k$ time slots, given that Node $A$ became active at time slot 1 is

$$
\sum_{i=1, j \neq f_{A(i)}}^{F} \pi_{1 j}\left(1-\frac{1}{F}\right)^{k-1}
$$

Now consider that Node $B$ was already transmitting the second packet of a message at $t=1$ when Node $A$ starts transmitting the first packet of its message. In this case, there are two possible configurations for Node $B$ 's transmission sequence as shown in Figure 3; once Node $B$ finishes transmitting the first message, it can become idle with a probability of $1-p_{B}$ (as shown in Config. 1), or it could immediately go into transmitting the first packet of a second message with a probability of $p=p_{B}$ (Config. 2). Since Node $A$ is assumed to be always transmitting on $F=1$ in this illustrative example, these states which should not be realized by Node $B$ to avoid a collision are shown with a darker shade. The resulting conditional probability that Node $A$ 's message does not collide with Node $B$ in any time slots, given that Node $A$ became active at time slot 1 is

$$
\sum_{i=1, j \neq f_{A(i)}}^{F} \pi_{2 j}\left[p\left(1-\frac{1}{F}\right)^{k-1}+(1-p)\left(1-\frac{1}{F}\right)^{k-2}\right]
$$

As we develop on this logic and consider Node $B$ in the process of sending the $n$-th packet of a message when Node $A$ became active, the possible set of configurations increases and the expression for the conditional success probability gets difficult. The possible configurations for $n=3$ for Node $B$ at $t=1$ are shown in Figure 4.

Using Eqs. (2) and (3), and following the steps outlined above, we can write down the expression for the conditional probability of no collision in multi-packet $(k \geq 2)$ S-Aloha system between Nodes $A$ and $B$ during $A$ 's active duration, $p_{n c_{(A, B)}}$, in a generic form as

$$
\begin{aligned}
p_{n c_{(A, B)}}= & \pi_{0}\left[\sum_{n=1}^{k-1} p(1-p)^{n}\left(1-\frac{1}{F}\right)^{k-n}+(1-p)^{k-1}\right](8) \\
& +(F-1) \pi_{i j} \sum_{n=1}^{k}\left[(1-p)^{n-1}\left(1-\frac{1}{F}\right)^{k-n}\right. \\
& \left.+\sum_{l=0}^{\max (n-2,0)} p(1-p)^{l}\left(1-\frac{1}{F}\right)^{k-l-1}\right], \quad k \geq 2
\end{aligned}
$$

Therefore, given the homogeneity across different nodes, using Eqs. (2), (3), and (8), we obtain the unconditional probability that a node's message does not collide with any other $M-1$ nodes as

$$
P_{s}=F \pi_{i j}\left(p_{n c_{(A, B)}}\right)^{M-1}=\frac{p\left(p_{n c_{(A, B)}}\right)^{M-1}}{[1-p(1-k)]}, k \geq 1
$$

Notice that for the special case of $F=1$, we get back the expression obtained in Eq. (4) for the single frequency case, and when $k=1$, we get $P_{s}=p\left(1-\frac{p}{F}\right)^{M-1}$ as expected.

\section{B. Heterogeneous Nodes}

Following Eq. (8), in the case of a system with heterogeneous nodes, the conditional probability of no collision between two nodes, $t$ and $m$, during $t$ 's active duration is

$$
\begin{aligned}
p_{n c_{(t, m)}}= & \pi_{0}\left[\sum_{n=1}^{k-1} p_{m}\left(1-p_{m}\right)^{n}\left(1-\frac{1}{F}\right)^{k-n}+\left(1-p_{m}\right)^{k-1}\right] \\
& +(F-1) \pi_{i j} \sum_{n=1}^{k}\left[\left(1-p_{m}\right)^{n-1}\left(1-\frac{1}{F}\right)^{k-n}\right. \\
& \left.+\sum_{l=0}^{\max (n-2,0)} p_{m}\left(1-p_{m}\right)^{l}\left(1-\frac{1}{F}\right)^{k-l-1}\right], k \geq 2
\end{aligned}
$$

Thus, the unconditional success probability for a message from Node $t$ becomes

$$
\Rightarrow P_{s}(t)=\frac{p_{t} \prod_{m=1, m \neq t}^{M} p_{n c_{(t, m)}}}{\left[1-p_{t}(1-k)\right]}
$$

Let $X_{t}$ be the indicator random variable that determines the message transmission success or failure of Node $t$, then

$$
\begin{aligned}
X_{t} & =1 \text { w.p. } P_{s}(t) \\
& =0 \text { w.p. } 1-P_{s}(t)
\end{aligned}
$$

Although $X_{t}$ are dependent random variables, because of the additivity of expectation, we have $\frac{1}{M} E(\bar{X})=$ $\frac{1}{M} \sum_{m=1}^{M} E\left(X_{t}\right)$. Hence, the unconditional probability of successful message transmission for any node is $P_{s}=$ $\frac{1}{M} \sum_{m=1}^{M} P_{s}(t)$, which gives

$$
P_{s}=\frac{1}{M} \sum_{m=1}^{M} \frac{p_{t} \prod_{m=1, m \neq t}^{M} p_{n c_{(t, m)}}}{\left[1-p_{t}(1-k)\right]}
$$




\section{System Throughput and Load}

To calculate the throughput or load of such a system of nodes (cluster), we first calculate the expected activity period of a node. On average, the expected number of slots for which a node is inactive is given by

$$
E(m)=\sum_{m=1}^{\infty} m p_{m}\left(1-p_{m}\right)^{m-1}=\frac{1-p_{m}}{p_{m}}
$$

So for every active $k$ time slots during which a node is transmitting a message, there is an inactive period of $\frac{1-p_{m}}{p_{m}}$ slots. A message transmitted is transmitted in $k+\left(1-p_{m}\right) / p_{m}$ slots on average. The conditional probability of a successful message transmission is given by may be defined by $\frac{P_{s}}{\pi_{i j}}$. Therefore, the throughput of a node is given by

$$
\text { Throughput }=\frac{k\left(P_{s} / \pi_{i j}\right)}{k+\left(1-p_{m}\right) / p_{m}}
$$

The load of the entire cluster of nodes is

$$
\text { Load }=\frac{M\left(1-\pi_{0}\right)}{F}=\left(\frac{M}{F}\right) \delta
$$

where $M / F$ captures the "group" size in terms of nodes sharing the same frequencies, and $\delta$ accounts for their activeness.

\section{RESUlTS}

In this section, we use the analytical expressions obtained in Section IV to illustrate some key results and numerical findings. In particular, we consider the question of "grouping" the POPs into MAC subnets and assigning the available frequencies to these subnets so as to improve throughput. For example, given $M=8$ nodes and $F=4$ frequencies, is the throughput higher in a "larger" group where all the nodes share all the available frequencies or "smaller" groups with a subset of nodes sharing a subset of frequencies. For this study we restrict ourselves to non-overlapping groups only, although the analytical results presented in Section IV can be used to analyze more generic cases. This allocation decision depends not only on the ratio of $\frac{M}{F}$ but also on the message length in packets and the system load, which is governed by the parameter $p$. For the analytically tractable case of single packet messages (i.e., $k=1$ ), we provide several results in Propositions 5.1-5.3 and then consider numerical examples for more general cases.

Proposition 5.1: For a given set of $M$ nodes and $F$ frequencies, smaller groups are better for low values of $p$ (i.e., $p \rightarrow 0)$.

Proof: Consider two groups, indexed by $i \in 1,2$, with a fixed ratio of nodes and frequency allocation, $r=M_{i} / F_{i}$. The throughput is $p\left(1-\frac{p}{F_{i}}\right)^{r F_{i}-1}$. For $F_{2}>F_{1}$, the difference in throughput of the two groups $\left(M_{2}=r F_{2}, F_{2}\right)$ and $\left(M_{1}=\right.$ $\left.r F_{1}, F_{1}\right)$ in the limit $p \rightarrow 1$ (and neglecting the higher orders) is $p^{2}\left(\frac{1}{F_{2}}-\frac{1}{F_{1}}\right)<0$. Hence, a smaller group of size $(M=$ $r, F=1$ ) is better for low values of $p$.

The result of the above proposition can be seen in Figures 5(a) and 5(b) which show the node throughputs as a function of the load for scenarios where the ratio of nodes to frequencies
$r=M / F$ is 2 and 4 , respectively. In particular, higher throughput is achieved with smaller groups when the load is low because partitioning resources reduce the likelihood of collision across nodes. For example, Figure 5(a) shows that for low values of $p$ (i.e. less load), the throughput achieved with "smaller" groups (i.e., say 16 distinct groups with 2 nodes sharing 1 frequency) is higher, but for higher values of $p \rightarrow 1$, "larger" groups (i.e., all 32 nodes sharing all the 16 frequencies) can be better. Proposition 5.2 quantifies the latter observation regarding high load scenarios.

Proposition 5.2: For a fixed ratio of $M / F$ i.e. $r=\frac{M}{F}$, it is better to form larger groups for high values of $p$ (i.e., $p \rightarrow 1$ ), if $r \geq 2$, and smaller groups when $r<2$.

Proof: When $p=1$, we have the node throughput as $T=$ $\left(1-\frac{1}{F}\right)^{r F-1}$. We find that $T(F)$ is a continuously increasing function (i.e., $\frac{\partial T}{\partial F} \geq 0$ ) only if

$$
r \geq \frac{1}{F+\left(F^{2}-F\right) \ln \left(1-\frac{1}{F}\right)} \Rightarrow 2-\frac{2}{3 F}+o\left(\frac{1}{F}\right)
$$

The previous expression has two important properties: (a) saturates to a value of 2 as $F \rightarrow \infty$, and (b) it is monotonically non-decreasing in $F$.

To see the latter property, consider its derivative w.r.t. $F$, which gives $\frac{-(2+(2 F-1) \ln (1-1 / F))}{\left[F+\left(F^{2}-F\right) \ln (1-1 / F)\right]^{2}}$. The derivative is positive $\forall F$ because $2+(2 F-1) \ln (1-1 / F) \leq 0 \forall F$ :

$$
\begin{aligned}
& (2 F-1)\left(1 / F+1 / 2 F^{2}+1 / 3 F^{3}+\ldots\right) \geq 2 \\
& =\sum_{m=2}^{\infty} \frac{m-1}{m(m+1) F^{m}}>0 \forall F
\end{aligned}
$$

These properties of the function $\frac{1}{F+\left(F^{2}-F\right) \ln \left(1-\frac{1}{F}\right)}$ imply that for $r>2, \frac{\partial T}{\partial F} \geq 0$, thus favoring the formation of larger groups.

Alternatively, $\frac{\partial T}{\partial F} \leq 0$ if $r<\frac{1}{F+\left(F^{2}-F\right) \ln \left(1-\frac{1}{F}\right)}$, then it is always better to form smaller groups. To see this, consider the largest possible value of $r<2$, which is $(2 F-1) / F$ (since $F$ and $M$ are integers). We now show that $r=(2 F-1) / F<$

$$
\begin{aligned}
\frac{1}{F+\left(F^{2}-F\right) \ln \left(1-\frac{1}{F}\right)} \text { because } \\
F+\left(F^{2}-F\right) \ln \left(1-\frac{1}{F}\right)-\frac{1}{2}\left(1-\frac{1}{2 F}\right)^{-1} \\
\Rightarrow \sum_{m=2}^{\infty} \frac{1-m}{2 m(m+1) F^{m-1}} \leq 0 \quad \forall F
\end{aligned}
$$

Hence, for $r=(2 F-1) / F$, the throughput will decrease with $F$, i.e., smaller groups are preferred.

Proposition 5.3: For $r \geq 2$, any two integer numbers $F_{2}=$ $c_{2} \geq F_{1}=c_{1}$, and a given ratio $r=\frac{M_{2}}{F_{2}}=\frac{M_{1}}{F_{1}} \geq 2$, the node throughput is higher in smaller groups, $\left\{M_{1}=r c_{1}, F_{1}=c_{1}\right\}$, when network load is low (i.e., $p \rightarrow 0$ ), and higher in larger groups, $\left\{M_{2}=r c_{2}, F_{2}=c_{2}\right\}$, when load is high (i.e., $p \rightarrow 1$ ).

Proof: This proposition mostly follows from the previous two. For low values of $p$, the throughput for a smaller group, $\left\{M_{1}=r c_{1}, F_{1}=c_{1}\right\}$, is higher than for a larger group, $\left\{M_{2}=r c_{2}, F_{2}=c_{2}\right\}$, i.e., $T_{1}>T_{2}$. When $p$ is high, then for $r \geq 2$, the throughput for the larger group is higher 


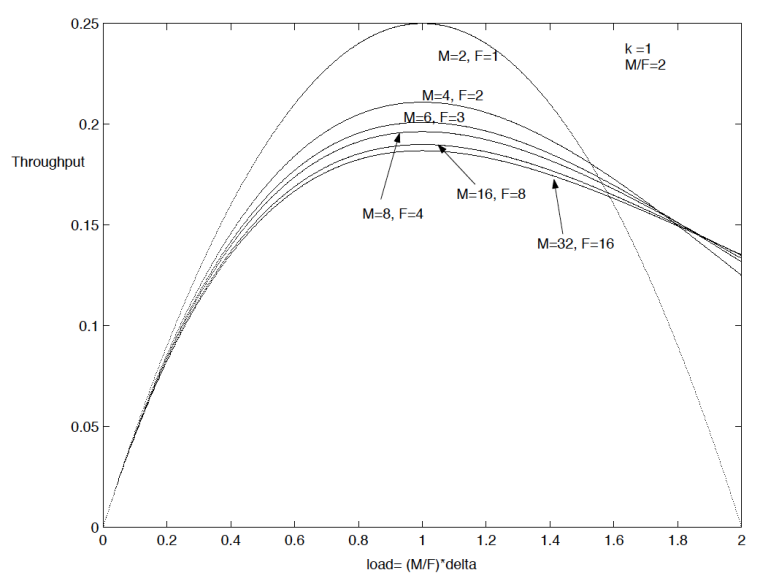

(a) Node throughput for $k=1$ and $M / F=2$ groups

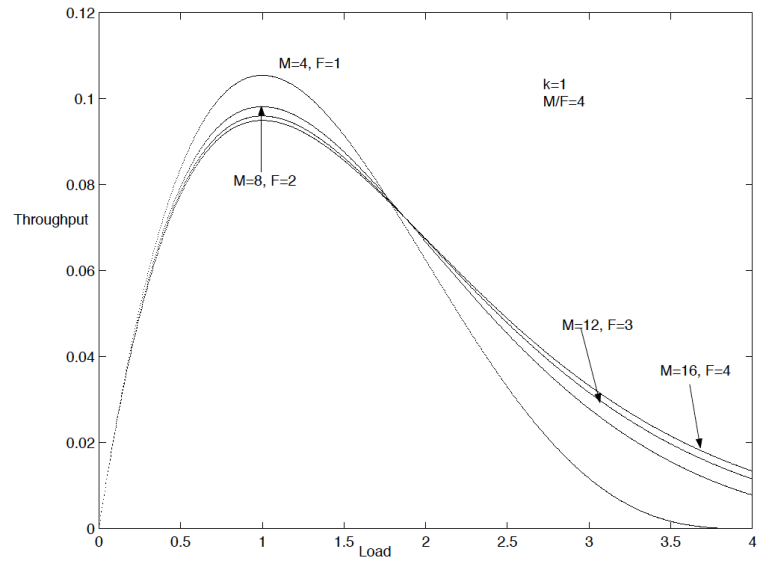

(b) Node throughput for $k=1$ and $M / F=4$ groups

Fig. 5. Smaller (larger) groups have higher throughput at lower (higher) loads

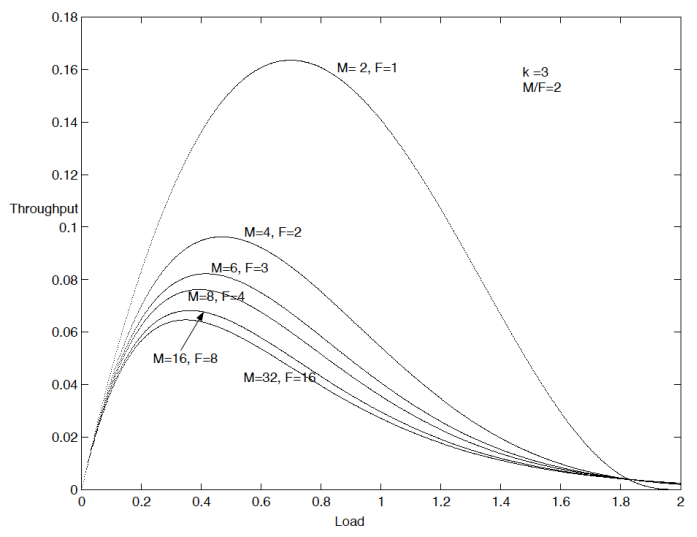

Fig. 6. Node throughput for $k=3$ and $M / F=2$ groups

and the throughput curves intersect. For $r<2$, then the throughput curves do not intersect, i.e., smaller groups have higher throughput.

Next, we consider the case of multi-packet messages. Figure 6 provides the graph for node throughput for varying load conditions for multi-packet messages with $k=3$. As before, we observe that the benefits of channel diversity may be lost due to random access in larger groups, and hence, smaller groups may indeed be more desirable in such scenarios.

These propositions and illustrations show that the choice of this group size is very much determined by the level of load in the system. This observation demonstrates that whether the availability of channel diversity is beneficial or not depends on several factors, including network parameters like $M / F, p, k$ etc. The closed form expressions presented earlier can thus be useful in determining the efficient partitioning of nodes and frequencies for maximizing system throughput.

\section{CONCLUSION}

This paper studies the issue of partitioning sensor nodes and frequencies to groups in order to maximize the system throughput. We model a deployment scenario of low cost sensor devices that operate in a basic random access mode. We provide closed form expressions for the throughput in a very generic setting of a multi-packet, p-persistent S-Aloha system with finite number of nodes and frequencies. The formulation is then used to show that the correct partitioning of nodes and frequencies can vary depending on the load of the system. This work can therefore help in better planning and allocation of network resources into logical subsets of sensor nodes.

There are several interesting lines for future extension of this work. In this work we have not modeled the impact finite arrival and that of retransmission policies, such as selective ARQ etc, but these are of particular interest as future work. Understanding the effect of packet coding on the throughput and partitioning is another direction for the next step.

\section{REFERENCES}

[1] D. Raychaudhuri, "ALOHA with Multipacket Messages and ARQ-Type Retransmission Protocols- Throughput Analysis", IEEE Transactions on Communications, Vol. 32, no. 2, pp. 148-154, February 1984.

[2] A. Myers and S. Basagni, "Wireless media access control", Handbook of Wireless Networks and Mobile Computing, John Wiley \& Sons, Inc. New York, NY, USA, 2002.

[3] R. T. B. Ma, V. Mishra, and D. Rubenstein, "An analysis of generalized slotted-Aloha protocols", IEEE/ACM Transactions on Networking, Vol. 17, Issue 3, June 2009.

[4] N. Abramson, "The ALOHA system- Another alternative for computer communications", Proc. of AFIPS, Vol. 37, pp. 281-285, 1970.

[5] M. J. Ferguson, "An approximation analysis of delay for fixed and variable length packets in an unslotted ALOHA channel", IEEE Trans. Commun., vol. 25, pp. 644-654, July 1977.

[6] S. Bellini and F. Borgonovo, "On the throughput of an ALOHA channel with variable length packets", IEEE Trans. Commun., vol. 28, pp. 1932 1935, Nov. 1980.

[7] R. Al-Naami and M. Al-Meer, "Performance of Slotted ALOHA with Multi-packet Buffers", Proc. of IEEE Tencon, pp. 669-673, 1992.

[8] G. Benelli, "Some new retransmission strategies for multipacket slotted ALOHA protocol", IEEE Proceedings, vol. 135(6), pp. 585-593, 1988.

[9] A. Carleial and M. Hellman, "Bistable behavior of Aloha-type systems", IEEE Trans. Commun., vol. COM-23, pp. 401-410, 1975.

[10] A. MacKenzie and S. Wicker, "Stability of multipacket slotted Aloha with selfish users and perfect information", INFOCOM, pp. 1583-1590, 2003.

[11] R. T. B. Ma, V. Mishra, and D. Rubenstein, "Modeling and analysis of generalized slotted-Aloha MAC protocols in cooperative, competitive, and adversarial environments", Proc. of ICDCS, July 2006.

[12] E. Vergetis, R. Guérin, and S. Sarkar, "Realizing the benefits of userlevel channel diversity", ACM SIGCOMM Computer Communication Review, Vol. 35, Issue 5, pp. 17-28, October 2005. 